\title{
In-house development of an FPGA-based MCA8K for gamma-ray spectrometer
}

\author{
Dang Lanh ${ }^{1 *}$, Pham Ngoc Son ${ }^{1}$ and Nguyen An Son ${ }^{2}$
}

\begin{abstract}
The objective of this work is domestic development of electronics instruments. It used for measuring ionization radiation and practical training at Nuclear Research Institute (NRI), Dalat, Vietnam. The aim of this work is to study and develop a novel MCA8k for Gamma-ray spectrometer concerning experimental nuclear physics. An approach for design and construction of the aforementioned instrument is to apply logic integrating techniques via Field Programmable Gate Arrays (FPGA) under Max + Plusll, Altera. The instrument allows interfacing to PC with self-developed application software. Scientific significance of this work is partly to contribute to opening a research direction in the field of nuclear electronics science for design and construction of radiation measurement instruments with the advanced IC technology in Vietnam. Practical significance of this work is partly to contribute to enhancement of capabilities in developing radiation measurement instruments for experimental research as well as practical training in nuclear physics. The advantages of FPGA: overcoming ballistic deficit, decrement of serial and parallel noise, flexible in programming, control of the system by software without an interfere of hardware. The disadvantages of FPGA: requirement of good knowledge of VHDL and professional tools for development of a expected project. A new electronics module of MCA8k has been achieved. Some main results obtained from the experimental testing are as follows: differential nonlinearity (DNL) of FPGA-MCA8k approximately $1.27 \%$, integral nonlinearity $(\mathrm{INL})=0.607 \%$, time conversion $\approx 2.2 \mu \mathrm{s}$, deadtime $(\mathrm{DT})$ is $0.75 \%$. Data Acquisition Program MCANRI written in $\mathrm{VC}^{++} 6.0$, self-executed under Windows XP environment.
\end{abstract}

Keywords: Field Programmable Gate Arrays (FPGA); Differential nonlinearity (DNL); Integral nonlinearity (INL)

\section{Introduction}

So far, FPGA can be used in four main areas: digital signal processing, $\mu \mathrm{C}$ integration, interfacing among the entity classes and reconfiguration of design. Recently, the technological development of a new generation of electronics circuits and its role in application designs always show many highlights. The advantages of digital systems for gamma-ray spectrum in comparison with those of conventional electronics systems are reflected in the ability executing complex algorithms for signal processing (Redus 2009). According to this approach, the highest quality of measurements achieving at both low and high count rates as using different radiation detectors is possible. The main functions of a spectrometer as filtering and amplifying signals, detecting and eliminating overlapped pulses, analysis of amplitude and emission of energy spectrum (CAEN

\footnotetext{
* Correspondence: lanhdng@yahoo.com

${ }^{1}$ Nuclear Research Institute, 01 Nguyen Tu Luc, Dalat, Vietnam

Full list of author information is available at the end of the article
}

2008), (Los Arcos and Garcia-Torano 1994) can be implemented effectively by digital algorithms using FPGA. The operations greatly increase the flexibility of the system, allow re-configuring and calibrating parameters without interfering the hardware (Bolic and Drndarevic 2002). In addition, the developed system can interface to PC easily. Recently, FPGA is used to develop nuclear instruments. These instruments are designed for high precision $\gamma$-ray spectroscopy with HPGe detectors and directly compatible with scintillator/PMT combinations: NaI, CsI, BGO, etc. As known, The DGF Pixie-4 is a multichannel data acquisition system for nuclear physics and other applications requiring coincident radiation detection (XIA LLC 2009), or the work for development of a fast flash ADC for nuclear spectroscopy system (Hien and Toshihiko 2001), or the issue for development of an FPGA based coincident system (Khang et al. 2013). Owing to a flexible inner structure in defining and changing the functions of electronics circuits by programming, FPGA expresses its capability for 


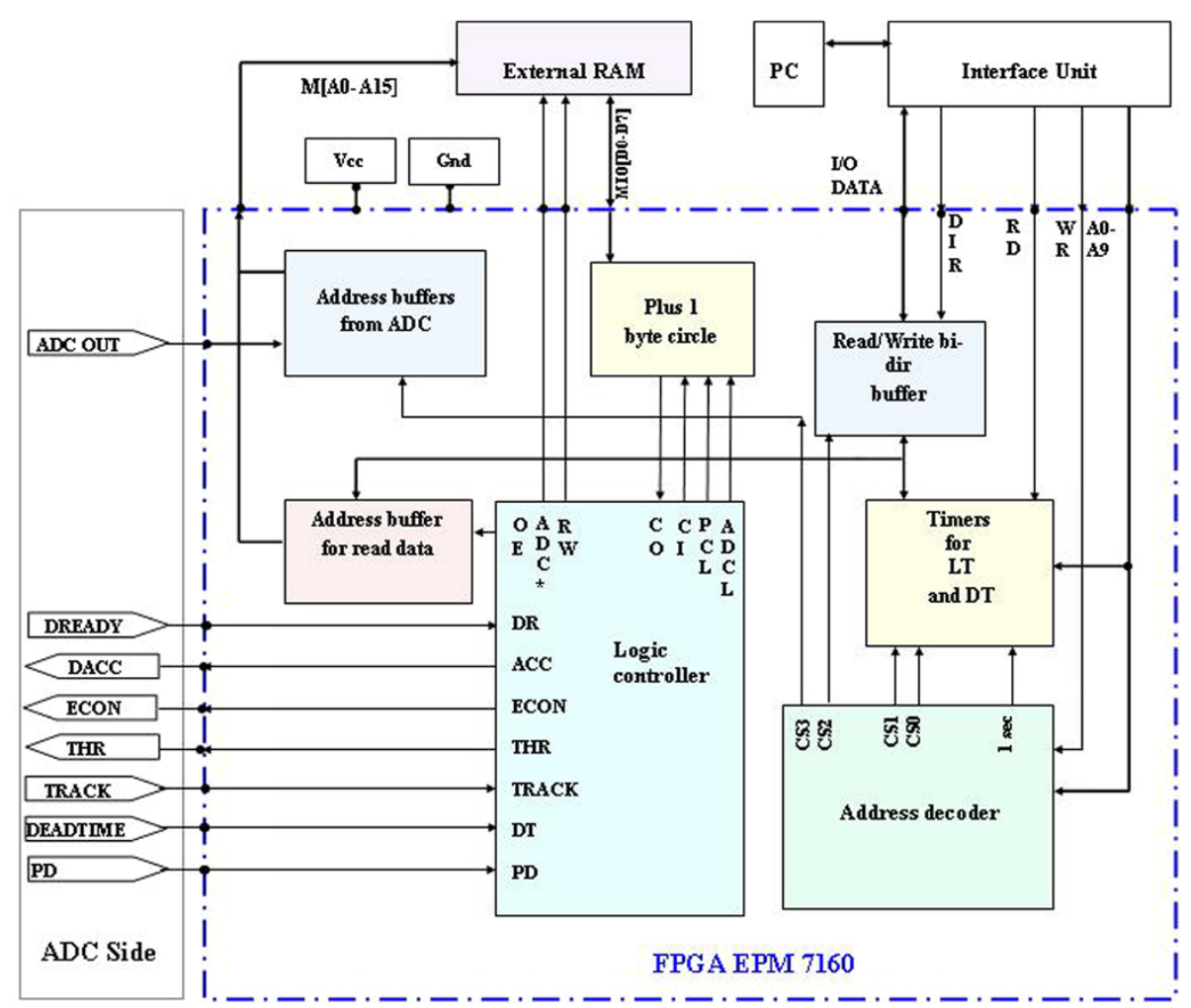

Figure 1 A block diagram of FPGA-MCA8k configuration.

applying in gamma ray spectroscopy. Logic-logic linking method using IC FPGA in Max + Plus II environment with EPM7160E was spread in main procedures: forming a project and the initial conditions of design, handling project, generating information of graphic interface, compiling and loading data into a specific architecture. As results, FPGA contains the entire contents of the design and operates as a micro controller. In this work, the instrument is carried out by a logic element linking method. To develop nuclear electronics instruments under the direction of FPGA, a

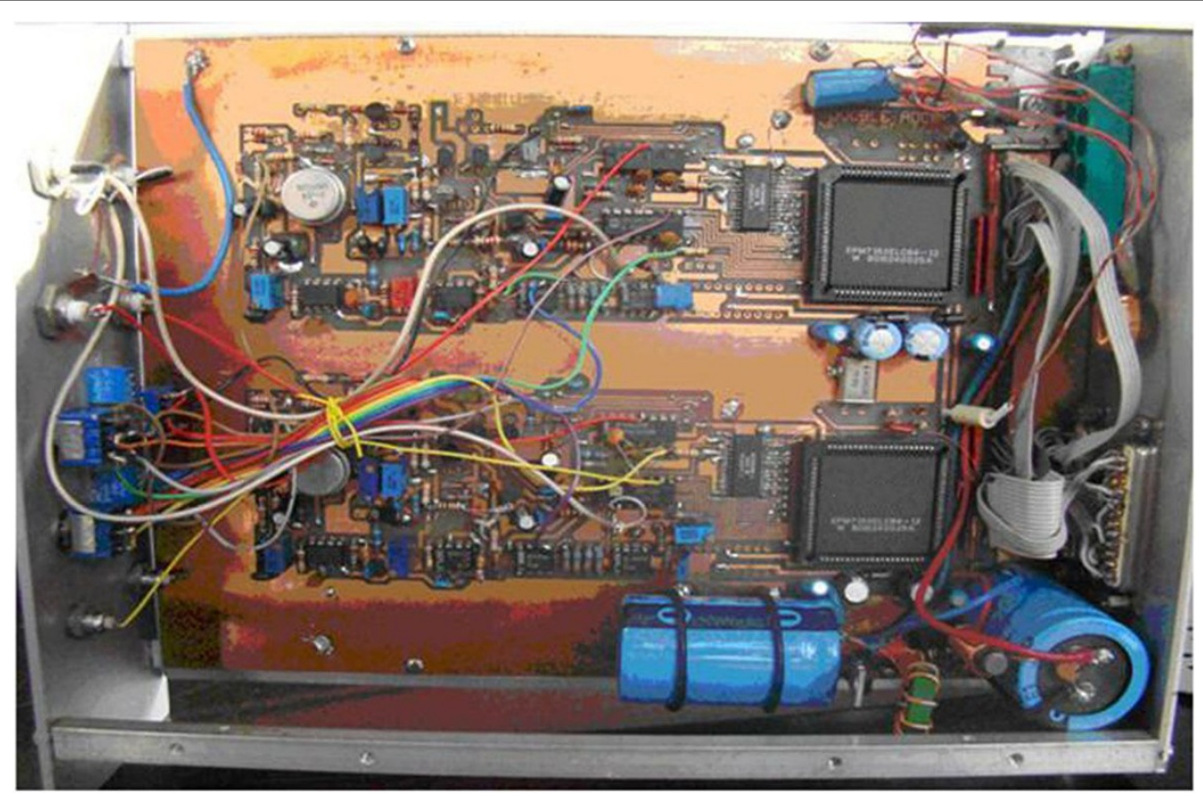

Figure 2 Layout of FPGA-ADC8k. 


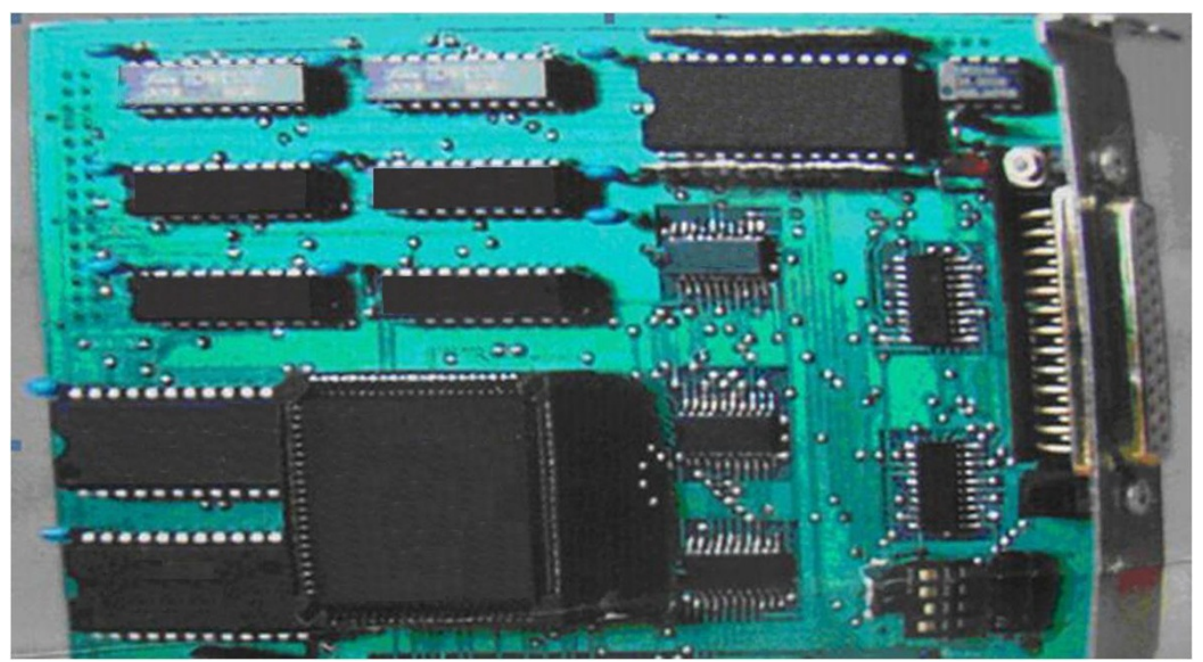

Figure 3 Layout of FPGA-MCD8k.

desired combination of hardware and software for performing algorithms of digital signal processing is current needs. Therefore, the following parts are presented: design and construction of MCA-8 k hardware, development of an application software for data acquisition and control of the instrument. On the other hand, the constructed instrument is able to interface to $\mathrm{PC}$ via a transferringreceiving port between peripheral devices and PC.

\section{Design and method}

\subsection{Logic-logic combination method for integrated} circuits of functional parts

A block diagram of MCA (Multi Channel Analyzer) is presented in Figure 1. It consists of two main parts: ADC part and MCD (Multi Channel Data processing) part. Layout of FPGA-ADC8k in Figure 2 is ADC part. It includes the following main stages: input voltage follower (used LH0032G), pulse stretcher, peak detection and track/hold sample, analog to digital conversion (used AD7899BR-1), one FPGA EPM7160 for logic control as well as logic control. Layout of FPGA-MCD8k in Figure 3 is MCD part. It includes digital input buffers, digital output buffers, an external memory SRAM, 2 timers, and also one EPM7160 for logic control. Logic-logic combination method for integrated circuits of two parts is explained as follows. ADC part accepts a signal, converts it to a digital number, this number called a digital copy from the original analog signal. The copy will be carried out by MCD part. All of

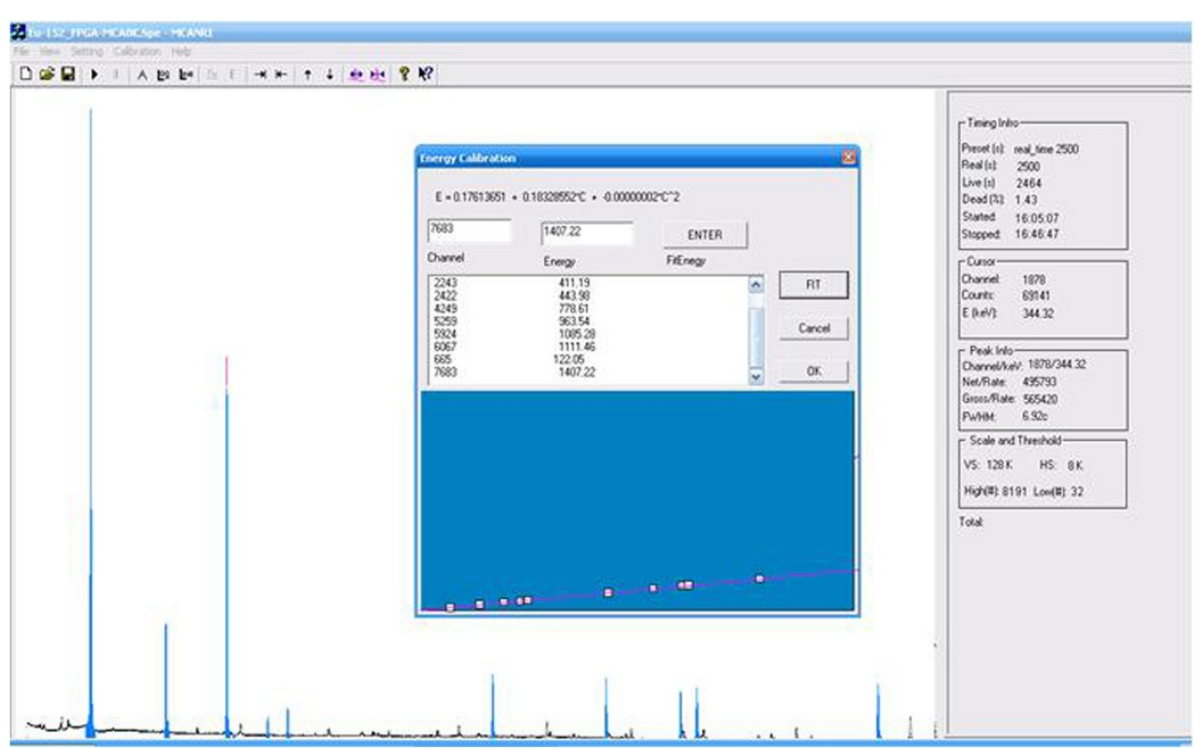

Figure 4 The interface page of MCANRI program. 
components for performance of electronics functions in MCD are formed inside an FPGA device, covered by a blue dash-line called the entity of the FPGA.

The instrument is constructed by FPGA application in which a logic processor is IC EPM7160E (a device of MAX 7000 family) with fast logic-logic combination about 5 ns (ALTERA 1998). The approach for setting data into FPGA entity was the professional method via parallel link. IC EPM7160E plays a role of internal logic controlling and data processing through all non-compact components. There are I/O ports allowing the FPGA to transfer external signals by each transaction either binary digits or TTL signals. In this study, the entity includes the following function elements: (i) A logic controller is responsible for the exchange of the question-response signals between MCD and ADC. (ii) Threshold conditions, peak detection, data output, and chip selection are carried out through a series of needed activities. As the asking-response

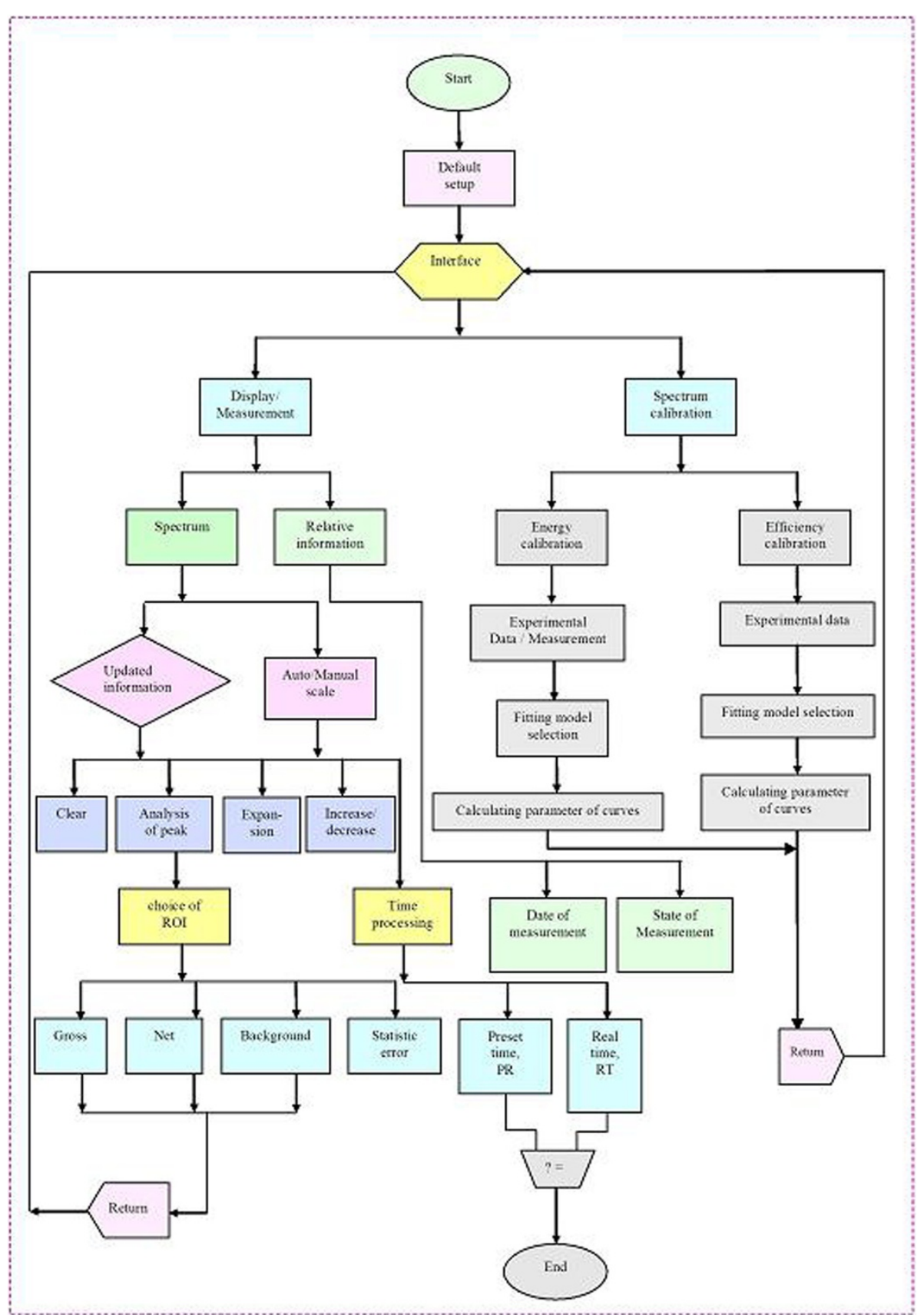

Figure 5 The algorithm flowchart of MCANRI program. 


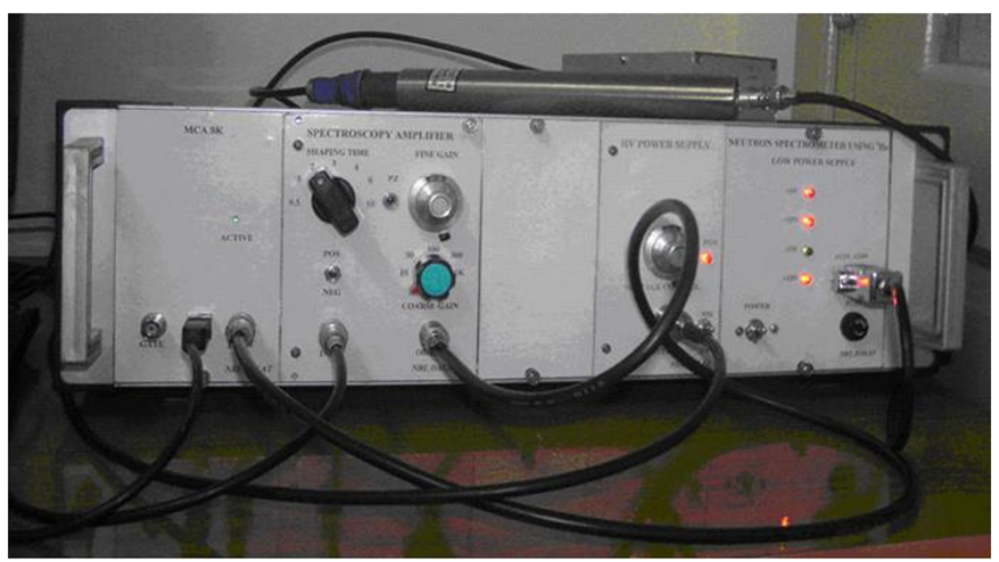

Figure 6 The neutron counting system used at the horizontal channel of the reactor, NRI, Dalat.

condition between ADC side and MCD side following a clock sharing rule is satisfied, a conversion process starts and stores data into the external SRAM.

The main task of FPGA EPM7160E is integration of all digital parts as well as logic control parts for the both parts of $\mathrm{ADC}$ and MCD in the designed project carried out by integrated software environment (ISE) Max + PlusII. Since the particularity of the EPM7160E family is entirely digitized, all the analog components could not be embedded inside it. When the linear input follower is satisfying the threshold conditions for the question-response logic, a peak is detected and passed to the logic controller within the FPGA for handling, and SRAM contains spectral information. In principle, DPRAM can be created in an FPGA, but SRAM has to be used because the capacity of
EPM7160E is not large enough. All other IC components are integrated in FPGA entity. Therefore, EPM7160E FPGA acts as the central data processor controlling the operation of the circuits by application software.

\subsection{The processing unit and operation of the MCA8k}

$\mathrm{I} / \mathrm{O}$ ports of the entity allow the FPGA communicating with outside signals in every transaction. A logic controller is responsible for the exchange of the question-response signals between MCD and ADC. When DR (data ready) gives MCD a ready status to collect data, MCD returns two pulses (ACC (accepted) and ECON (enable conversion)) to $\mathrm{ADC}$ with variable thresholds establishing differentiated window for carrying out data acquisition. As the track signal (peak detection) appears, dead-time (DT) pulse allows

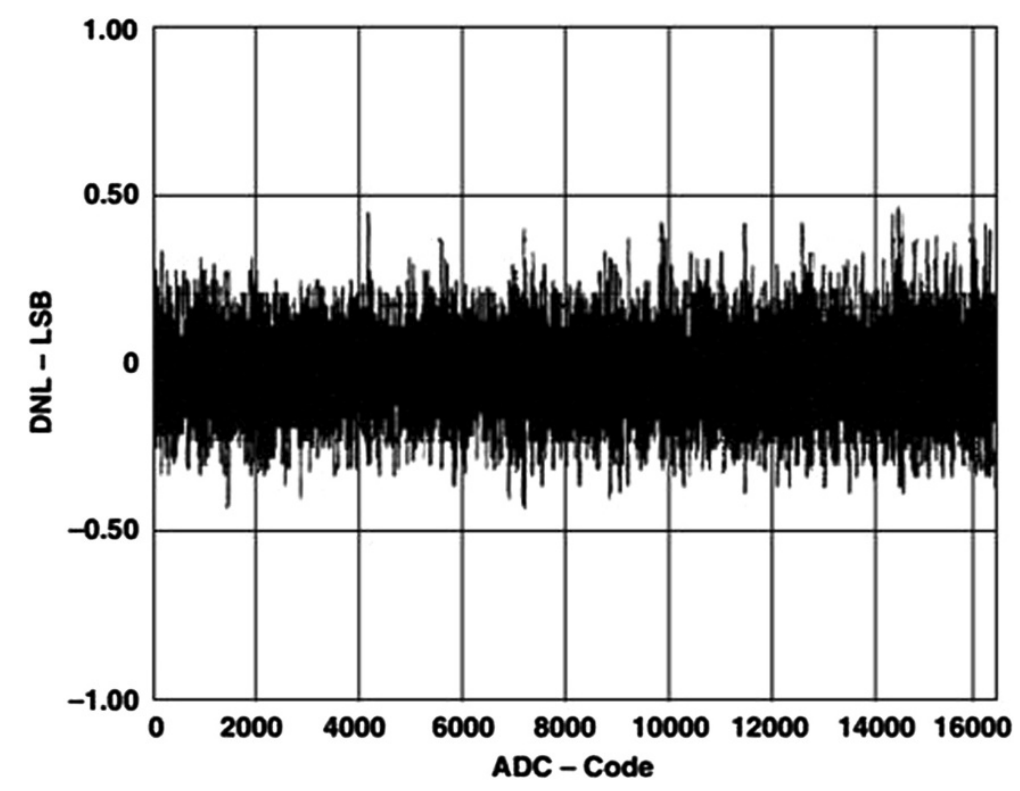

Figure 7 Typical DNL plot for the AD7899. 


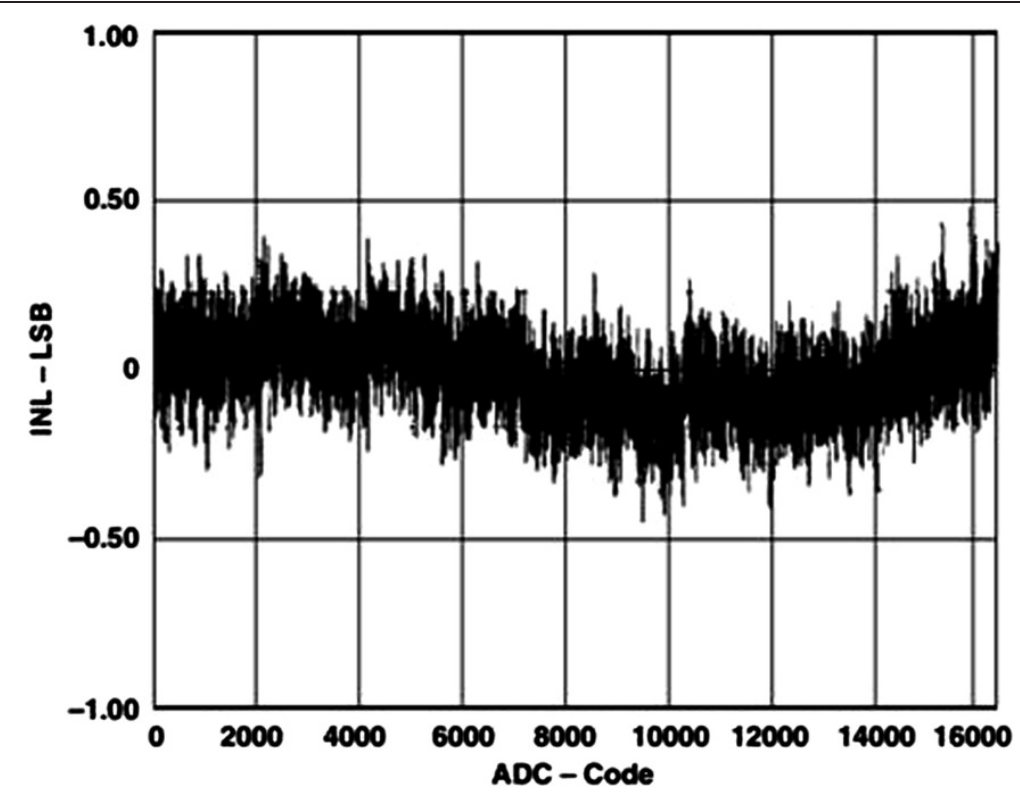

Figure 8 Typical INL plot for the AD7899.

the logic controller sending a pulse to the FPGA for timestamp (as a percentage). In parallel with the signal recognition logic and the question-and-response interface, the memory read/write function, living time (LT), dead time (DT) and real-time (RT) are done via other control logic pulses. To locate the address for SRAM write cycle, ADC pulse opens two byte buffers of 13-bit address. Peak amplitude content (reflecting energy information) existing in DR will be written on each cell of SRAM memory when OE (open enable) is low and RW (read-write) is high. Existing data on the local exchange line MIO [D0 - D7] written by each byte data via a series of internal logic control pulses ADCL (ADC latch), CI (Carry input), CO
(Carry output) and $\mathrm{ADC}^{*}$ (ADC ative low). To download the content contained in the SRAM, a memory read cycle is carried out by detecting low and high address with the decoded signal CS3. When two bytes of address read cycle have positioned SRAM, then contents will be read out via CS2. Data receivertransmitters from peripheral to $\mathrm{PC}$ via bidirectional buffer the signal selected port (read or write) PSEL and choose the direction DIR signal effect. timers is programmable (2 bytes each) will be calculated from DT, LT clocks by 1 second. FPGA EPM7160E acts as the central data processor controlled the operation of the instrument by MCANRI.exe software applications.

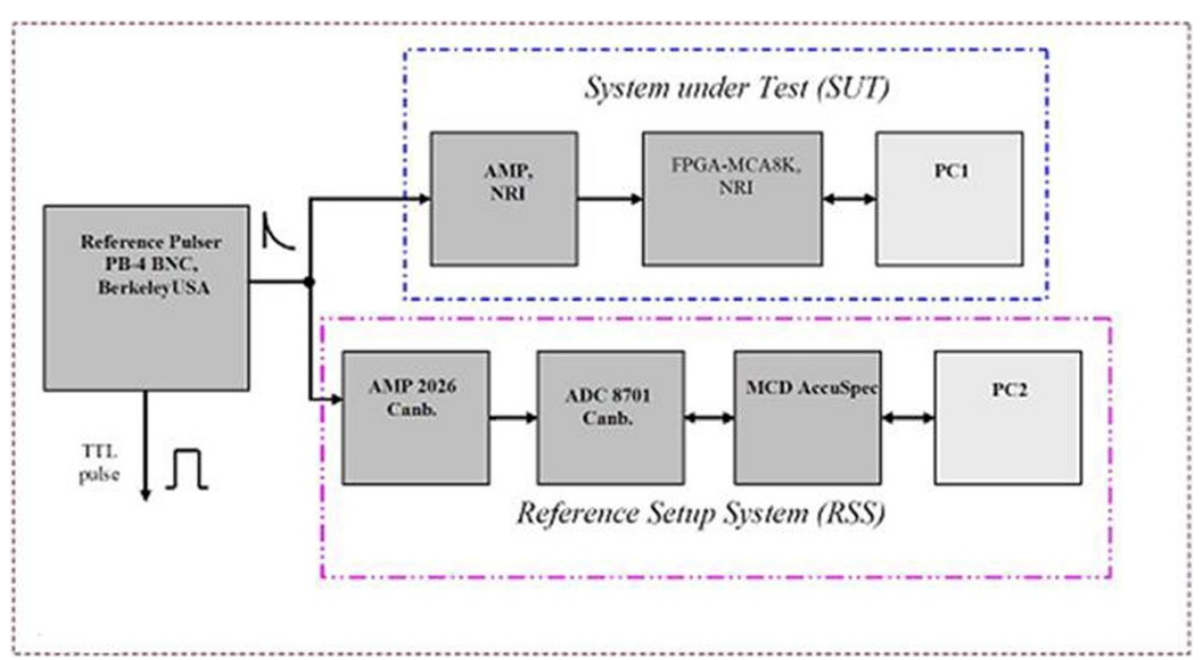

Figure 9 Configuration of measurement for differential nonlinearity of MCA. 


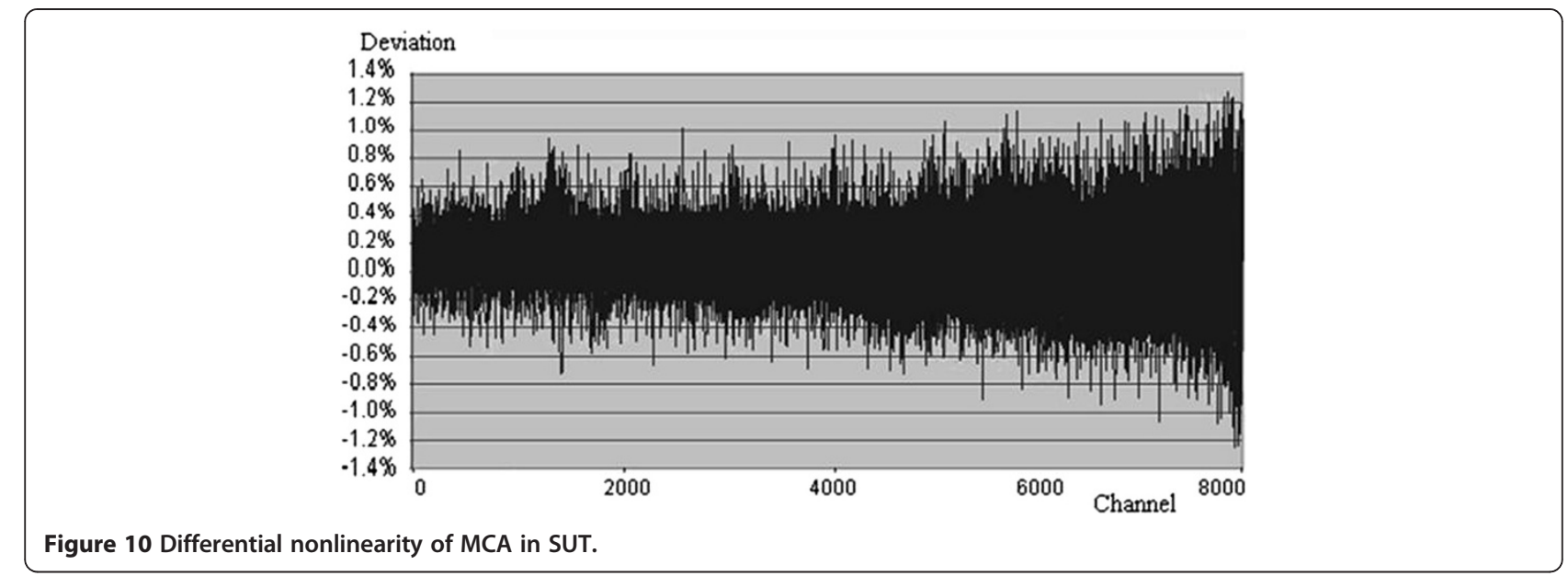

\subsection{The application software}

For controlling of data acquisition, setting of parameter and interfacing with user, an application program was developed, named MCANRI. The basic functions of the program are storing information of spectrum in PC memory devices, setting measuring parameters, and calibrations. The interface page and the algorithm flowchart are presented in Figures 4 and 5.

When the program begins, all default icons are displayed on the console screen. Two main branches of the flowchart are for measurement with peak analysis and energy/efficiency calibrations. As satisfying a combination of the instrument, spectrum and timing information are displayed. The spectrum is displayed and updated every millisecond in auto scale or manual mode.

Besides, the application program MCANRI is not only used for data acquisition in gamma spectrometer, but also used for the neutron counting system using ${ }^{3} \mathrm{He}$ detector at horizontal experiment channels of the reactor, Nuclear Research Institute (NRI), Dalat. Figure 6 shows the neutron counting system.

\section{Experimental measurements and results}

\subsection{Determination of differential nonlinearity of MCA}

One of the largest contributions to the DNL/INL of the FPGA-MCA system is coming from the ADC. As constructing the system, AD7899 from ANALOG DEVICES (Analog Devices, Inc. 2001) is used. The AD7899 is a fast, low-power, 14-bit A/D converter that operates from a single $5 \mathrm{~V}$ supply. The part contains a $2.2 \mu \mathrm{s}$ successive-approximation ADC, a track/hold amplifier, $2.5 \mathrm{~V}$ reference, on-chip clock oscillator, signal conditioning circuitry, and a high-speed parallel interface. The part accepts analog input ranges of $\pm 10 \mathrm{~V}$. The plots in Figures 7 and 8 show typical DNL and INL for the AD7899, respectively.

To test differential nonlinearity of MCA (DNL FPGA-MCA $_{\text {), }}$, an experimental arrangement is performed as shown in Figure 9. This configuration consists of two independent measurement branches: the upper branch of the system (system under test - SUT) is to test the technical characteristics of the instruments formed from AMP-NRI (Hien and Lanh 1988), FPGA-MCA8k, computer 1, data

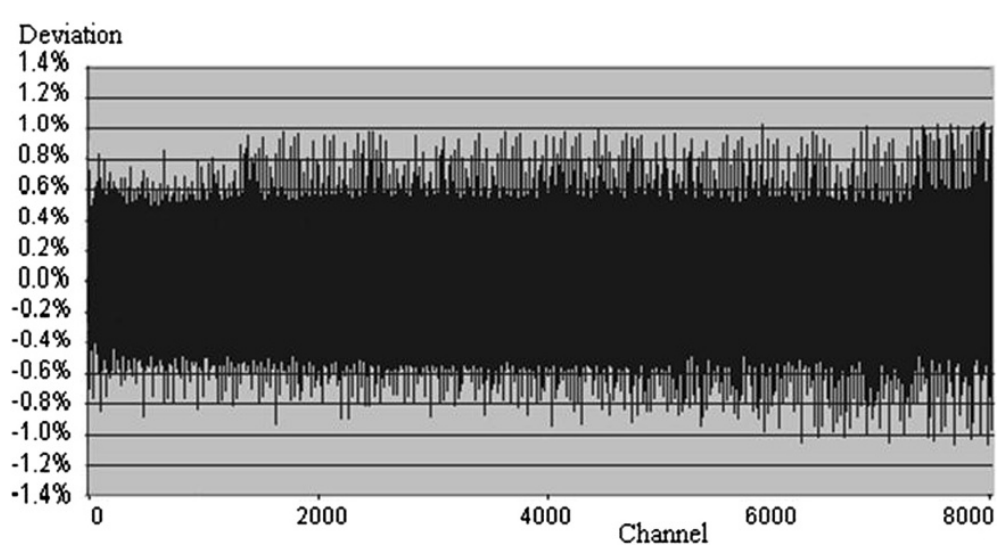

Figure 11 Differential nonlinearity of MCA in RSS. 
Table 1 Experimental results of the integral nonlinearities of MCAs

\begin{tabular}{lcccccccc}
\hline Instruments & $\mathbf{T}_{\text {meas. }}(\mathbf{s})$ & $\mathbf{V}_{\text {in }}(\mathbf{m V})$ & Mode & $\boldsymbol{\tau}_{\text {AMP }}(\boldsymbol{\mu s})$ & Range (\#) & Counts & DT (\%) & DNL (\%) \\
\hline RSS $_{\text {Accuspec }}$ & 36000 & $10^{4}$ & PHA & 4 & 8192 & 179154 & 0.47 & 1.03 \\
SUT $_{\text {FPGA-8K }}$ & 36000 & $10^{4}$ & PHA & 4 & 8192 & 178972 & 0.56 & 1.27 \\
\hline
\end{tabular}

collection program MCANRI; the below branch (reference setup system - RSS) includes AMP 2026 Canberra, ADC 8701 Canberra, MCD Accuspec V1.1, software MCA Series 100 and computer 2. Saw-generator type LG-1 BNC, Berkeley, USA controls signal amplitude in random pulser DB-2 BNC, Berkeley, USA. Shaping time of both AMPs is chosen $4 \mu$ s to reduce the effect of rising time as using the reference pulser. The experiment gives counts for full range of 8192 channels: $\sum_{i=1}^{8192} x_{i}=1474389136$, leading to the average number of counts: $\mathrm{N}_{\mathrm{av}} \approx 179979.143$. From $\mathrm{N}_{\mathrm{av}}$ to find the maximum deviation value in 8192 channels: $\Delta \mathrm{N}_{\max }=$ $\left(\mathrm{N}_{\mathrm{x}}-\mathrm{N}_{\mathrm{av}}\right)_{\max }=2285.735$. Thus, differential nonlinearity (IAEA 2008) of the FPGA-MCA8k is: DNL ${ }_{\text {FPGA-MCA8K }}=$ $(2285.735 / 179979.143) \times 100 \% \approx 1.27 \%$. Statistical fluctuation of counts in Figure 10 represents its differential nonlinearity for SUT. Similar to the above-mentioned calculation, differential nonlinearity obtained in RSS (please refer to Figure 11): $\mathrm{DNL}_{\mathrm{RSS}}=1.03 \%$. Also, deadtime in both systems, respectively: $\mathrm{DT}_{\mathrm{FPGA}-\mathrm{MCA} 8 \mathrm{~K}}=0.56 \%$ and $\mathrm{DT}_{\mathrm{RSS}}=0.47 \%$. All results are shown in Table 1 .

In principle, both of results from RSS and SUT must be the same. However, this result is different from the other. SUT's result is worse than that of RSS. Please refer to Figures 10 and 11. It is clear that the quality of designed circuits of SUT is not better than that of RSS. Because statistical fluctuation of counts in higher channels is bigger than statistical fluctuation of counts in lower channels, therefore deviations of counts in higher channels must be larger. This reason makes spectrum in higher channels bigger, it means that there is an increment for DNL.

When the specific technical parameters of the MCA are tested, a reference setup system (RSS) as a basis for evaluating operating modes as well as the reliability of the execution method demonstrated through experimental data measurements. Table 1 showed that the deviation between differential nonlinearity of FPGA-MCA8k and that of MCD Accuspec is $|(1.03-1.27)| / 1.03=0.233$. In comparison with differential nonlinearity of MCD-Accuspec, differential nonlinearity of FPGA-MCA8k is larger than that of MCD-Accuspec $(1.27+0.233)-1.03=0.473$. Although differential nonlinearity of FPGA-MCA8k does not achieve the nominal standard, it is still acceptable for radiation measurements and training purpose. In the reality, it affects the channel widths in analog to digital conversion, and variation of widths leads a high fluctuation of counts.

\subsection{Determination of integral nonlinearity of MCA}

To test integral nonlinearity of MCA (INL FPGA-MCA $_{\text {), an }}$ experimental configuration is performed in Figure 12. Two measurement programs are MCANRI and MCA Series 100. Reference Pulser PB-4 BNC, Berkeley, USA generates a positive nuclear tail pulse with rise time of $25 \mathrm{~ns}$ and falling time of $50 \mu \mathrm{s}$. Set thresholds of LLD $\approx 16 \mathrm{mV}$, ULD maximum of $10000 \mathrm{mV}$. Changing the incremental steps from 0 to $10000 \mathrm{mV}$, test steps are 40. The corresponding channel-voltage value pairs listed in the Table 2 . From the experimental fitting function of $y=0.82254 x-12.3832$, where $\mathrm{x}$ is the amplitude of the input signal, $\mathrm{y}$ is the estimated channel, -12.3830 is the amplitude at channel zero, 0.82250 is the slope of the fit line and coefficient of

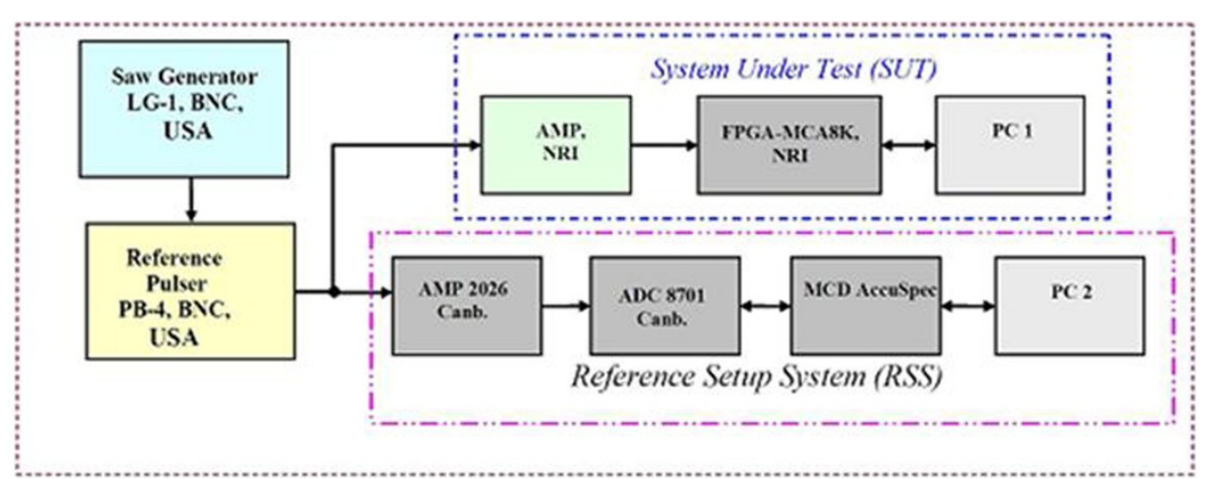

Figure 12 Configuration of measurement for integral nonlinearity of MCA. 
Table 2 Results of integral nonlinearity of the MCA

\begin{tabular}{|c|c|c|c|c|c|c|c|c|c|}
\hline No. & Voltage $X_{i}(m V)$ & $Y_{r}$ & $Y_{i}$ & $\Delta \mathrm{Y}_{\max }$ & No. & Voltage $(\mathrm{mV})$ & $Y_{r}$ & $Y_{i}$ & $\Delta Y_{\max }$ \\
\hline 1 & 17 & 18 & 1.59998 & 16.40002 & 21 & 4962 & 4089 & 4068.329 & 20.67068 \\
\hline 2 & 191 & 158 & 144.7219 & 13.27806 & 22 & 5219 & 4259 & 4279.624 & -20.62444 \\
\hline 3 & 431 & 327 & 342.1315 & -15.13154 & 23 & 5469 & 4501 & 4485.164 & 15.83556 \\
\hline 4 & 690 & 568 & 556.0618 & 11.9382 & 24 & 5707 & 4659 & 4680.839 & -21.83852 \\
\hline 5 & 930 & 745 & 753.3802 & -8.3802 & 25 & 6032 & 4956 & 4948.041 & 7.95948 \\
\hline 6 & 1188 & 971 & 965.4975 & 5.50252 & 26 & 6270 & 5149 & 5143.715 & 5.2854 \\
\hline 7 & 1440 & 1199 & 1172.682 & 26.3182 & 27 & 6484 & 5293 & 5319.657 & -26.65684 \\
\hline 8 & 1699 & 1388 & 1385.621 & 2.37876 & 28 & 6749 & 5576 & 5537.529 & 38.47076 \\
\hline 9 & 1938 & 1584 & 1582.117 & 1.88252 & 29 & 7002 & 5767 & 5745.536 & 21.46428 \\
\hline 10 & 2190 & 1817 & 1789.302 & 27.6982 & 30 & 7342 & 6042 & 6025.07 & 16.92988 \\
\hline 11 & 2450 & 2001 & 2003.063 & -2.0634 & 31 & 7584 & 6127 & 6224.033 & -97.03284 \\
\hline 12 & 2696 & 2219 & 2205.315 & 13.68524 & 32 & 7973 & 6574 & 6543.853 & 30.14692 \\
\hline 13 & 2950 & 2408 & 2414.143 & -6.1434 & 33 & 8242 & 6727 & 6765.014 & -38.01412 \\
\hline 14 & 3202 & 2456 & 2621.328 & -165.3277 & 34 & 8522 & 7034 & 6995.219 & 38.78108 \\
\hline 15 & 3452 & 2851 & 2826.868 & 24.13228 & 35 & 8700 & 7102 & 7141.563 & -39.5634 \\
\hline 16 & 3703 & 3023 & 3033.23 & -10.22988 & 36 & 9162 & 7532 & 7521.401 & 10.59868 \\
\hline 17 & 3965 & 3298 & 3248.636 & 49.3642 & 37 & 9320 & 7659 & 7651.303 & 7.6974 \\
\hline 18 & 4216 & 3467 & 3454.998 & 12.00204 & 38 & 9497 & 7798 & 7796.825 & 1.17508 \\
\hline 19 & 4485 & 3661 & 3676.159 & -15.159 & 39 & 9837 & 8114 & 8076.359 & 37.64068 \\
\hline 20 & 4716 & 3879 & 3866.078 & 12.92204 & 40 & 9872 & 8132 & 8105.135 & 26.86508 \\
\hline
\end{tabular}

determination $R^{2}=0.9997$ (please refer to Figure 7), replacing $X_{i}=(17 \div 9872)$ with $i \in[1,40]$, then 40 values of $\mathrm{Y}_{\mathrm{i}}$ is obtained. Therefore, $\Delta Y_{\max }=\left(\mathrm{Y}_{r}-Y_{i}\right)_{\max }=49.36$ and $Y_{\text {max }}=8132$. According to reference (ORTEC, CAMAC ADCs and Memories), integral nonlinearities in SUT and RSS are, respectively, calculated as follows:

$$
\begin{aligned}
& I N L_{F P G A-M C A 8 k}=\frac{\Delta Y_{\max }}{Y_{\max }} \cdot 100 \%=\frac{49.3642}{8132} \cdot 100 \%=0.607 \%, \\
& I N L_{M C D-\text { Accuspec }}=0.15 \% .
\end{aligned}
$$

The Integral nonlinearity $\mathrm{INL}_{\mathrm{FPGA}-\mathrm{MCA}} \mathrm{SK}_{\mathrm{K}}$ is about 4 times bigger than $\mathrm{INL}_{\mathrm{MCD} 8 \mathrm{~K}-\mathrm{Accuspec}}$; therefore, integral nonlinearity of the instrument is worse as higher energy. On the other hand, as presented in Figure 13, the signal amplitude is strong correlation with the corresponding channel within statistical fluctuation. It could be estimated that electronics noise and/or unstable baseline restorer are the basic reasons of the difference between the MCA8k and the standard one. In other word, DNL/INL affect the channel width distributions and uncertainties of counts in the system. As results, the higher the energy, the worse the resolution.

3.3. Test of count accuracy and data throughput of the MCA Accuracy of count numbers of FPGA-MCA8k was determined by an experimental configuration in Figure 9 using TTL pulse output. Frequency generator is changed in a range from $f_{\min }=90 \mathrm{~Hz}$ to $f_{\max }=300 \mathrm{kHz}$, for both of RSS and SUT systems, measuring in PHA mode with preset time $t_{p r}=10000 \mathrm{~s}$. The deviation of RSS and SUT was calculated (CANBERRA 2009) by the

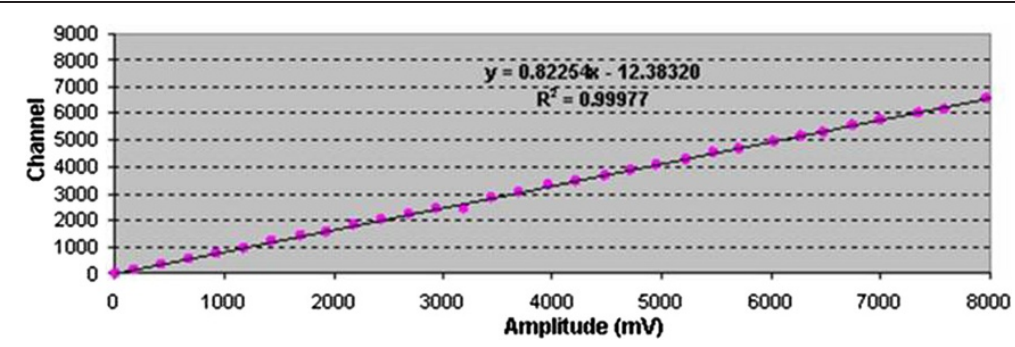

Figure 13 Integral nonlinearity of the MCA. 
Table 3 Accumulated counts versus time and their differences between two systems

\begin{tabular}{cccccc}
\hline Test times & Time of measurement & Frequency & Counts, $\boldsymbol{C}_{\mathbf{r}}$, in RSS & Counts, $\mathbf{C}_{\mathbf{t}}$, in SUT & Deviation D\% (\%) \\
\hline 1 & $\mathrm{t}_{\mathrm{pr}}=10000 \mathrm{~s}$ & $\mathrm{f}_{\min }=90 \mathrm{~Hz}$ & 899075 & 898526 & 0.0611 \\
2 & $\mathrm{t}_{\mathrm{pr}}=10000 \mathrm{~s}$ & $\mathrm{f}=500 \mathrm{~Hz}$ & 4975124 & 4973392 & 0.0348 \\
3 & $\mathrm{t}_{\mathrm{pr}}=10000 \mathrm{~s}$ & $\mathrm{f}=1 \mathrm{kHz}$ & 9938031 & 9930125 & 0.0796 \\
\hline
\end{tabular}

formula: $D \%=\frac{\left|C_{r}-C_{i}\right|}{C_{r}} \cdot 100 \%$, where $C_{r}$ is counts obtained in RSS and $C_{t}$ is counts in SUT. When elapsed time is equal to preset time $t_{p r}$, both systems automatically stop. Accumulation of counts against time and their differences between two systems are shown in Table 3.

In Table 3, test results in terms of counting accuracy and frequency of input-output data of the present designed MCA show the average deviation of 0.06 in the frequency range of $90 \mathrm{~Hz}-1 \mathrm{kHz}$, in comparison with the standard case.

\subsection{Test of spectrum calibration and peak analysis}

A spectrometer for measuring gamma ray emitted from ${ }^{152} \mathrm{Eu}$ source was setup in Figure 14. C2019 type HPGe detector, Inter-techniques is fed to all functional electronics modulars: AMP 2026-Canberra, FPGAMCA8k, HV 5 k- NRI, and PC. Application software for getting data is MCANRI. All parameters setup with MCANRI and timing information are as follows:

$$
\begin{aligned}
& { }^{152} \text { Eu source: } \mathrm{T}_{1 / 2}=13.51 \text { years, Activity A }(\mathrm{Bq}) \\
& =123238 \text {; }
\end{aligned}
$$

HPGe C2019 detector, Intertechniques: positive HVPS = $2500 \mathrm{~V}$, resolution $2.05 \mathrm{keV}$ at peak $1332.5 \mathrm{keV}$ of ${ }^{60} \mathrm{Co}$, $\mathrm{t}_{\text {real }}=2500 \mathrm{~s}, \mathrm{LL}=32, \mathrm{UL}=8191$.

When $t_{\text {live }}=t_{\text {preset }}=2500$ seconds, MCANRI program automatically stops; ${ }^{152} \mathrm{Eu}$ spectrum displays on screen in Figure 4. It allows user performing all following procedures: Energy calibration using quadratic curve, peak area calculation P, gross G, background B, and standard deviation $\sigma_{\mathrm{p}}$. Refer to Figure 4, all following information are derived: preset time is equal to real time being
$2500 \mathrm{~s}$, elapsed time is $2464 \mathrm{~s}$, dead time is $1.43 \%$; starting at 16:05:07 and stopping at 16:46:47; the main pointer at central energy $344.32 \mathrm{keV}$ with 69141 counts according to channel 1878. Lower level is 32, upper level is 8191 , vertical scale is $128 \mathrm{k}$, range of measurement is $8 \mathrm{k}$. As entering 10 experimental 'channel-energy' values and fitting, the experimental quadratic energy calibration curve $(\mathrm{keV})$ is a function of channel $(C)$ :

$$
\mathrm{E}(k e V)=0.1761+0.1832_{*} \mathrm{C}-0.00000002_{*} \mathrm{C}^{2} .
$$

After calibrating energy, making ROI (region of interest) of peak is the next step; at that time, peak area, background, gross, standard deviation and energy resolution are calculated (IAEA 1991), respectively. ${ }^{152} \mathrm{Eu}$ spectrum and the energy calibration curve via a fit function of ten peaks with energy-channel value pairs is shown in Figure 4. Table 4 shows the experimental results.

\section{Conclusions}

This work carried out a design and construction of an FPGA-based MCA8k. FPGA EPM7160E acts as the central data processor controlled operations of the instrument by MCANRI software application. The integrated software environment for development of the entity FPGA is Max + PlusII, Altera. Main technical characteristics are as follows:

- FPGA-MCA8k interfacing to PC via the parallel port (LPT),

- Resolution: 8192 channels,

- Time conversion: approximate to $2.2 \mu \mathrm{s}$,

- The Integral nonlinear $\mathrm{INL}_{\mathrm{FPGA}-\mathrm{MCA} 8 \mathrm{~K}} \approx 0.607 \%$ of the full scale,

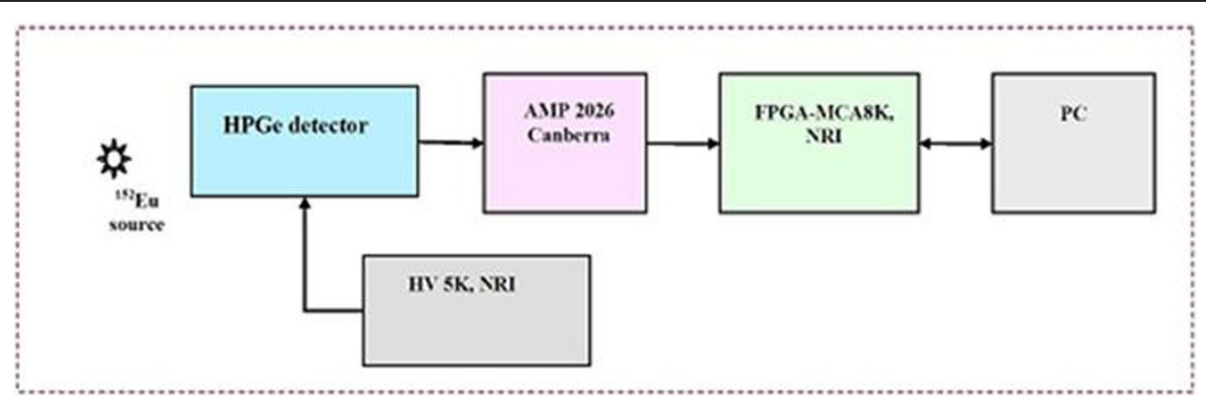

Figure 14 An experimental spectrometer for measuring gamma spectrum from ${ }^{152} \mathrm{Eu}$ source. 
Table 4 The quantitative values of ten-experiment gamma-ray energy peaks from ${ }^{152} \mathrm{Eu}$

\begin{tabular}{ccccccccc}
\hline $\begin{array}{c}\text { Gama-ray energy } \\
\text { from Eu-152 } \\
\mathbf{E}(\mathbf{k e V})\end{array}$ & $\begin{array}{c}\text { Emission } \\
\text { probility } \\
\mathbf{P}_{\mathbf{Y}}(\%)\end{array}$ & $\begin{array}{c}\text { MCANRI energy } \\
\text { calibrated } \\
\mathbf{E}(\mathbf{k e V})\end{array}$ & $\begin{array}{c}\text { FWHM } \\
\mathbf{( k e V )} \\
\text { for SUT }\end{array}$ & $\begin{array}{c}\text { FWHM } \\
\text { (keV) } \\
\text { for RSS }\end{array}$ & $\begin{array}{c}\text { Net area } \\
\text { of peak }\end{array}$ & $\begin{array}{c}\text { Measured } \\
\text { efficiency } \\
\text { (\%) }\end{array}$ & $\begin{array}{c}\text { Calibrated } \\
\text { fitting } \\
\text { efficiency (\%) }\end{array}$ & $\begin{array}{c}\text { Deviation } \\
\text { (Measured-Fitting) }\end{array}$ \\
\hline 121.78 & 28.58 & 122.05 & 1.11 & 1.07 & 908937 & 1.03 & 1.029 & 0.001 \\
244.69 & 7.58 & 245.01 & 1.24 & 1.19 & 176315 & 0.75 & 0.768 & -0.018 \\
344.28 & 26.50 & 344.50 & 1.27 & 1.25 & 495793 & 0.67 & 0.619 & 0.051 \\
411.11 & 2.234 & 411.19 & 1.54 & 1.50 & 35394 & 0.51 & 0.544 & -0.034 \\
443.96 & 3.148 & 443.98 & 1.65 & 1.59 & 49876 & 0.51 & 0.513 & -0.003 \\
778.90 & 12.942 & 778.79 & 1.68 & 1.63 & 139620 & 0.35 & 0.349 & 0.001 \\
964.07 & 14.605 & 963.54 & 1.84 & 1.81 & 144575 & 0.32 & 0.316 & 0.004 \\
1085.87 & 10.207 & 1085.28 & 1.91 & 1.86 & 100528 & 0.32 & 0.298 & 0.022 \\
1112.07 & 13.644 & 1111.48 & 1.99 & 1.92 & 114867 & 0.27 & 0.294 & -0.024 \\
1408.00 & 21.005 & 1407.22 & 2.10 & 2.07 & 156054 & 0.24 & 0.239 & 0.001 \\
\hline
\end{tabular}

- The Differential nonlinear DNL FPGA-MCA8K $_{\text {. }} \approx 1.27 \%$ over the measurement range,

- Maximum count capacity per channel is 16777215 ,

- The low and up levels for the ADC are controlled by software,

- Input receiving positive, unipolar pulse peak amplitude from 0 to $10 \mathrm{~V}$,

- Data Acquisition Program MCANRI written in $\mathrm{VC}^{++}$ 6.0, self-executing under Windows XP environment.

Field Programmable Gate Arrays (FPGA) technique has been successfully applied for development of a new MCA unit by means of logic-logic combination using the integrated software environment Max + plusII of Altera. This study creates a potential of low cost electronics module for gamma-ray spectrometers at Nuclear Research Institute, Dalat, Vietnam. In this study, we have designed, fabricated, tested and adjusted the new MCA module for technical parameters to meet requirements of practical applications. There are $\mathrm{I} / \mathrm{O}$ ports allowing the FPGA entity to transfer external signals by each transaction either binary digits or TTL signals. It includes the following function elements: (i) A logic controller is responsible for the exchange of the question-response signals between MCD and ADC. (ii) Threshold conditions, peak detection, data output, port selection, and chip selection are carried out through a series of needed activities. This MCA module is properly interfacing to PC through LPT or USB port. Regarding software, an application program called MCANRI has been developed by the $\mathrm{VC}^{++} 6$ compiler. This version of program contains almost basic functions required in radiation spectrum measurements, such as energy, efficiency calibrations, peak analysis, and timing parameter setup. In addition, it can be applied for data processing to test the technical characteristic parameters of the fabricated instrument and calculate the sufficient physics quantities in spectroscopy experiments measuring ionization radiation.

\section{Abbreviations}

ADC: Analog to digital converter; DC: Direct current; DIR: Direction; FWHM: Full-width half-maximum; I/O: Input/Output; LLD: Low level discrimination; MIO: Memory input-output; PCL: Port controlling link; PSEL: Port selection; RSS: Reference setup system; RW: Read-write; SRAM: Static random access memory; SUT: System under test; ULD: Upper level discrimination.

\section{Competing interests}

The authors declare that they have no competing interests.

\section{Authors' contributions}

DL carried out the research work; PNS carried out software programming, and NAS carried out analysis and tests. Three authors read and approved the final manuscript.

\section{Acknowledgments}

The author expresses grateful thanks to the Director, Nuclear Research Institute (NRI), Dalat, Vietnam for providing support and facility for carrying out this research project.

\section{Author details}

${ }^{1}$ Nuclear Research Institute, 01 Nguyen Tu Luc, Dalat, Vietnam. ${ }^{2}$ University of Dalat, 01 Phu Dong Thien Vuong, Dalat, Vietnam.

Received: 1 December 2013 Accepted: 18 October 2014 Published: 10 November 2014

\section{References}

ALTERA (1998) MAX+PLUS II Programmable Logic Development System \& Software, version 8.0

Analog Devices, Inc. (2001) 5 V Single Supply 14-Bit 400 kSPS ADC, AD7899

Bolic M, Drndarevic V (2002) Digital gamma-ray spectroscopy based on FPGA technology. NIM A 482:761-766

CAEN (2008) Digital Pulse Processing in Nuclear Physics, Revision 2.1 CANBERRA (2009) Genie 2000 Customization Tools Manual, Version 3.2

Hien DS, Lanh D (1988) Quasi-symmetrical triangular output spectroscopy amplifier. XIII International symposium on Nuclear electronics, Varna, Bulgaria, pp 353-355

Hien DS, Toshihiko S (2001) Development of a fast flash ADC for nuclear spectroscopy system. NIM A 457:356-360

IAEA (1991) X-ray and gamma ray standards for detector calibration, IAEA-TECDOC-619 
IAEA (2008) Test Procedure for Multichannel Analyzer Systems, Procedure № MRNI-514, REV. D0

Khang PD, Dien NN, Lanh D, Hai NX, Tuan PN, Hoa ND, Son NA (2013) A design configuration of an FPGA-based coincident spectrometry system. J Anal Sci Methods Instrum 3:158-162

Los Arcos JM, Garcia-Torano E (1994) A new digital pulse height analysis method for radiation spectroscopy. Nucl Instr Meth Phys Res A 339:99-101

ORTEC, CAMAC ADCs and Memories. http://www.ortec-online.com/ download/camac-adc-memory.pdf

Redus R (2009) Digital pulse processors: theory of operation, AN-DPP-001

XIA LLC (2009) User's Manual Digital Gamma Finder (DGF), DGF-4C Revision F, Version 4.03

doi:10.1186/2193-1801-3-665

Cite this article as: Lanh et al.: In-house development of an FPGA-based MCA8K for gamma-ray spectrometer. SpringerPlus 2014 3:665.

\section{Submit your manuscript to a SpringerOpen ${ }^{\circ}$} journal and benefit from:

- Convenient online submission

- Rigorous peer review

- Immediate publication on acceptance

- Open access: articles freely available online

- High visibility within the field

- Retaining the copyright to your article

Submit your next manuscript at $\gg$ springeropen.com 